\title{
A Novel Consensus Protocol for Two-Dimension First-Order Multiagent Systems
}

\author{
Yibo Zhang, Jinfeng Gao, and Jia Ren \\ Department of Automation, Zhejiang Sci-Tech University, Hangzhou, Zhejiang 310038, China \\ Correspondence should be addressed to Yibo Zhang; zhangy41@163.com
}

Received 15 January 2015; Accepted 9 March 2015

Academic Editor: Yun-Bo Zhao

Copyright (c) 2015 Yibo Zhang et al. This is an open access article distributed under the Creative Commons Attribution License, which permits unrestricted use, distribution, and reproduction in any medium, provided the original work is properly cited.

\begin{abstract}
A novel consensus protocol for two-dimension first-order multiagent systems (MAS) is proposed. After a novel protocol for twodimension first-order MAS is adopted; consensus and two kinds of possible results are concerned. Then consensus asymptotical conditions and ranges of key parameters are given, and convergence speed is analyzed. Numerical examples illustrate effectiveness of the proposed consensus protocol.
\end{abstract}

\section{Introduction}

Networks have been concerned for many years, and it has been researched in many areas such as in sensor networks [1], distribute networks, or wireless networks. Multiagent systems (MAS) have been concerned for many years. It reflects relationships of individuals in a network, such as critical rules or protocols or control of each individual [2]. One common way of pattern is that all agents converge to the same value, which is called consensus or agreement.

Consensus problem is applied in robots, spacecraft, and cooperative multiple vehicles, and it mainly concerns exchange ways of information. For example, reference [3] presented theoretical explanation for characters of continuous and discrete MAS with/without leaders. This classical work showed relations between connection of agents, protocols, and consensus. And [4] researched a new technique based on complex Laplacian to achieve arbitrary formation shape and global stability. Reference [5] mainly researched linear time-varying MAS, a necessary and sufficient condition of consensus ability with a set of admissible protocols. Then some applications are put up; [6] discussed a kind of autonomous scale control of multi-agent in a MAS with shape constrains. It provided a new method for choosing an appropriate geometry and discussed system effect by different parameters. Reference [7] considered MAS with leader of bounded unknown input. After two distributed discontinuous controllers were added, a sufficient condition was applied to ensure stabilization. References $[8,9]$ researched grouping control and coordinate control of MAS according in different conditions.

Suppose there are $n$ agents in MAS and they are connected by wireless networks, which can be described as a (directed) graph. The graph can be expressed in an adjacency matrix form called Laplacian matrix $L=\left[l_{i k}\right](1 \leq i, k \leq n)$ that satisfies the following conditions:

$$
\begin{gathered}
l_{i k} \leq 0 \quad i \neq k \\
\sum_{k=1}^{n} l_{i k}=0 \quad i=1,2, \ldots, n .
\end{gathered}
$$

According to [3], the following premise is introduced.

Premise. A first-order MAS should satisfy the condition that exchange topology has a spanning tree or $-L$ has only one zero eigenvalue and all the other nonzero eigenvalues have negative real parts.

The following is based on the fact that the premise is satisfied. In this work, a kind of two-dimension first-order MAS is concerned. Then a novel protocol and its consensus are proposed, and range of key parameter is given. Moreover, 
convergence speed is analyzed. Several simulations are shown to ensure the results.

\section{Consensus and Convergence Speed}

2.1. Consensus for Two-Dimension MAS. Suppose there are $n$ agents in a plat coordinate system, which means each agent has two coordinate positions named as $x$ and $y$, respectively. Dynamic equations of the $i$ th agent $(i=1,2, \ldots, n)$ are shown as following:

$$
\begin{aligned}
& \dot{x}_{i}=u_{x i} \\
& \dot{y}_{i}=u_{y i} .
\end{aligned}
$$

The consensus is that all agents finally converge together, with their positions converging to the same value, or it can be expressed as $\left|x_{i}-x_{k}\right| \rightarrow 0$ and $\left|y_{i}-y_{k}\right| \rightarrow 0$, when $t \rightarrow \infty$, where $i, k=1,2, \ldots, n$.

Protocol of two-dimension system is introduced as

$$
\begin{aligned}
& u_{x i}=-\sum_{k=1}^{n} l_{i k}\left[\left(x_{i}-x_{k}\right)+\gamma\left(y_{i}-y_{k}\right)\right] \\
& u_{y i}=-\sum_{k=1}^{n} l_{i k}\left[\left(x_{i}-x_{k}\right)+\left(y_{i}-y_{k}\right)\right] .
\end{aligned}
$$

Let $x=\left[\begin{array}{llll}x_{1} & x_{2} & \cdots & x_{n}\end{array}\right]^{T}$ and $y=\left[\begin{array}{llll}y_{1} & y_{2} & \cdots & y_{n}\end{array}\right]^{T}$. Equation (2) changes to the following form:

$$
\left[\begin{array}{c}
\dot{x} \\
\dot{y}
\end{array}\right]=\left[\begin{array}{l}
u_{x i} \\
u_{y i}
\end{array}\right]=\Gamma\left[\begin{array}{l}
x \\
y
\end{array}\right]
$$

where $\Gamma=\left[\begin{array}{cc}-L & -\gamma L \\ -L & -L\end{array}\right]$.

Suppose $\mu_{i}(i=1,2, \ldots, n)$ is the $i$ th eigenvalue of $-L$; then

$$
\operatorname{det}\left(\lambda I_{n}+L\right)=\operatorname{det}\left(\lambda I_{n}-(-L)\right)=\prod_{i=1}^{n}\left(\lambda-\mu_{i}\right)
$$

Because $-L$ has exactly one zero eigenvalue, and all the other nonzero eigenvalues have negative real parts; without loss of generality, let $\mu_{1}=0$ and the other nonzero eigenvalues $\mu_{i}=-\alpha_{i} \pm j \beta_{i}$, where $\alpha_{i}>0(i=2,3, \ldots, n)$. If $\mu_{i}$ is real, $\beta_{i}=0$, and $\beta_{i}>0$ if there are conjugate complex eigenvalues.

Let $\bar{\mu}_{i}(i=1,2, \ldots, 2 n)$ be the $i$ th eigenvalue of $\Gamma$; then $\operatorname{det}\left(\lambda I_{2 n}-\Gamma\right)=\prod_{i=1}^{2 n}\left(\lambda-\bar{\mu}_{i}\right)$.

Note that

$$
\begin{aligned}
\operatorname{det}\left(\lambda I_{2 n}-\Gamma\right) & =\operatorname{det}\left(\left[\begin{array}{cc}
\lambda I_{n}+L & \gamma L \\
L & \lambda I_{n}+L
\end{array}\right]\right) \\
& =\operatorname{det}\left(\lambda^{2} I_{n}+2 \lambda L+(1-\gamma) L^{2}\right) .
\end{aligned}
$$

Let $\lambda_{1}$ and $\lambda_{2}$ be roots of the character equation $\lambda^{2}+2 \lambda+$ $(1-\gamma)=0$; then there are two kinds of possibilities according to different $\gamma$.

(1) $\lambda_{1}$ and $\lambda_{2}$ Are Real. If $\gamma \geq 0, \lambda_{1}=1+\sqrt{\gamma}$, and $\lambda_{2}=1-\sqrt{\gamma}$ are real, then (6) can be decomposed to the following form:

$$
\begin{aligned}
\operatorname{det}\left(\lambda I_{2 n}-\Gamma\right)= & \operatorname{det}\left(\lambda^{2} I_{n}+2 \lambda L+(1-\gamma) L^{2}\right) \\
= & \operatorname{det}\left(\lambda I_{n}+(1+\sqrt{\gamma}) L\right) \\
& \cdot \operatorname{det}\left(\lambda I_{n}+(1-\sqrt{\gamma}) L\right) .
\end{aligned}
$$

Compared with (5), (7) turns to

$$
\begin{aligned}
\operatorname{det}\left(\lambda I_{2 n}-\Gamma\right)= & \operatorname{det}\left(\lambda I_{n}+(1+\sqrt{\gamma}) L\right) \\
& \cdot \operatorname{det}\left(\lambda I_{n}+(1-\sqrt{\gamma}) L\right) \\
= & \prod_{i=1}^{n}\left(\lambda-(1+\sqrt{\gamma}) \mu_{i}\right) \prod_{i=1}^{n}\left(\lambda-(1-\sqrt{\gamma}) \mu_{i}\right) .
\end{aligned}
$$

Then the following lemma is proposed.

Lemma 1. When $0 \leq \gamma<1$, consensus protocol (3) achieves consensus asymptotically, if and only if - L has exactly one zero eigenvalue and the other $n-1$ eigenvalues have negative real parts, which is equal to the condition if and only if $\Gamma$ has exactly two zero eigenvalues and the other $2 n-2$ eigenvalues have negative real parts.

Proof.

Sufficiency. If $0 \leq \gamma<1,1-\sqrt{\gamma}>0$, so it is obvious that $\bar{\mu}_{1}=(1-\sqrt{\gamma}) \mu_{1}=0$ and $\operatorname{Re}\left(\bar{\mu}_{i}\right)=-(1-\sqrt{\gamma}) \alpha_{i}<0$ $(i=2,3, \ldots, n)$. This means that $\bar{\mu}_{i}(i=1,2, \ldots, n)$ have the same sign with $\mu_{i}(i=1,2, \ldots, n)$. The same conclusion can be drawn when $\bar{\mu}_{i}=(1+\sqrt{\gamma}) \mu_{i}(i=1,2, \ldots, n)$ if $0 \leq \gamma<1$. Considering (8), the following conclusion is drawn: $\Gamma$ has exactly two zero eigenvalues and the others have negative real parts, which is equal to $-L$ having exactly one zero eigenvalue and the others have negative real parts.

Let $p=\left[\begin{array}{ll}p_{1}^{T} & p_{2}^{T}\end{array}\right]^{T}$ be an eigenvector of $\Gamma$ associated with one of zero eigenvalues, where $p_{1}, p_{2} \in R^{n}$; then

$$
\Gamma p=\left[\begin{array}{cc}
-L & -\gamma L \\
-L & -L
\end{array}\right]\left[\begin{array}{l}
p_{1} \\
p_{2}
\end{array}\right]=0,
$$

which implies that $-L p_{1}=0$ and $-L p_{2}=0$ if $0 \leq \gamma<1$. Note that $-L$ has only one linearly independent eigenvector associated with zero eigenvalue, so $p_{1}=p_{2}$. Moreover, $\Gamma$ has only one linearly independent eigenvector $p=\left[\begin{array}{ll}p_{1}^{T} & p_{1}^{T}\end{array}\right]^{T}$; that is, zero eigenvalue of $\Gamma$ has geometric multiplicity equal to one.

All of the above show that if $-L$ is chosen as asymptotically, $\Gamma$ achieves consensus asymptotically also.

Necessity (reduction to absurd). Suppose $\Gamma$ having exactly two zero eigenvalues and the other eigenvalues having 
negative real parts do not hold. From sufficient process, relationships between eigenvalues of $\Gamma$ and $-L$ are $\bar{\mu}_{i}=(1+$ $\sqrt{\gamma}) \mu_{i}$ or $\bar{\mu}_{i}=(1-\sqrt{\gamma}) \mu_{i}$, which means zero eigenvalues of $\Gamma$ are double times those of $-L$. Suppose there are more than two zero eigenvalues of $\Gamma$, for example, four zero eigenvalues, so there are at least two zero eigenvalues of $-L$.

According to [3], consensus cannot be achieved, which is contradicting with the premise, so there must be only two zero eigenvalues of $\Gamma$.

Then suppose there is at least one nonzero eigenvalue having positive real part. Note that $\Gamma$ can be transformed to Jordan canonical form by a nonsingular matrix $Q$ :

$$
\Gamma=Q^{-1} J Q
$$

Then

$$
\begin{aligned}
e^{\Gamma t} & =Q^{-1} e^{J t} Q \\
& =Q^{-1}\left[\begin{array}{ccc}
1 & 0 & 0_{1 \times(2 n-2)} \\
0 & 1 & 0_{1 \times(2 n-2)} \\
0_{(2 n-2) \times 1} & 0_{(2 n-2) \times 1} & e^{J^{\prime} t}
\end{array}\right] Q .
\end{aligned}
$$

It can be seen that if there is at least one eigenvalue with positive real part of $\Gamma$, when $t \rightarrow \infty$ and $e^{J^{\prime} t} \rightarrow \infty$, then $e^{\Gamma t} \rightarrow \infty$, which is contradicting with the following condition: "consensus protocol (2) achieves consensus asymptotically."

So the assumption is wrong.

(2) $\lambda_{1}$ and $\lambda_{2}$ Are Conjugate Complex Eigenvalues. If $\gamma<0$ and $\lambda_{1,2}=1 \pm j \sqrt{-\gamma}$ are one pair of conjugate complex eigenvalues, then (6) can be decomposed to the following form:

$$
\begin{aligned}
\operatorname{det}\left(\lambda I_{2 n}-\Gamma\right)= & \operatorname{det}\left(\lambda I_{n}+(1-j \sqrt{-\gamma}) L\right) \\
& \cdot \operatorname{det}\left(\lambda I_{n}+(1+j \sqrt{-\gamma}) L\right) \\
= & \prod_{i=1}^{n}\left(\lambda-(1-j \sqrt{-\gamma}) \mu_{i}\right) \\
& \cdot \prod_{i=1}^{n}\left(\lambda-(1+j \sqrt{-\gamma}) \mu_{i}\right) .
\end{aligned}
$$

Then the following lemma is proposed.

Lemma 2. When $-\min _{\mu_{i} \neq 0}\left(\alpha_{i}^{2} / \beta_{i}^{2}\right)<\gamma<0$, consensus protocol (3) achieves consensus asymptotically, if and only if $-L$ has exactly one zero eigenvalue and the other $n-1$ eigenvalues have negative real parts, which is equal to the condition if and only if $\Gamma$ has exactly two zero eigenvalues and the other $2 n-2$ eigenvalues have negative real parts.

The proof is omitted for similarity with Lemma 1. The differenence is range of $\gamma$, which is described as follows.

Because $\bar{\mu}_{i}=(1+j \sqrt{-\gamma}) \mu_{i}$ or $\bar{\mu}_{i}=(1-j \sqrt{-\gamma}) \mu_{i}$, if $\mu_{1} \neq 0$, two cases are concerned.
Case 1 (all nonzero eigenvalues $\mu_{i}(i=2,3, \ldots, n)$ are real). According to the premise, $\mu_{i}<0$, then both $\bar{\mu}_{i}=(1+j \sqrt{-\gamma}) \mu_{i}$ and $\bar{\mu}_{i}=(1-j \sqrt{-\gamma}) \mu_{i}$ have negative real parts.

Case 2 (there are at least one pair of conjugate complex eigenvalues). Suppose there is only one pair of conjugate complex eigenvalues marked as $\mu_{i+}$ and $\mu_{i-}$. Let $\mu_{i \pm}=-\alpha \pm j \beta$, where $\alpha>0$ and $\beta>0$. Because $\bar{\mu}_{i}$ is multiplied by two vectors $1 \pm \sqrt{-\gamma}$ and $\mu_{i}$, its module is product while phase angle is the sum of them. All possible results are shown in Figure 1.

In Figure $1, \theta_{i}$ denotes intersection angle between vector $\mu_{i \pm}$ and $Y$-axis and $\theta_{\Gamma}$ is abstract phase angle of $1 \pm j \sqrt{-\gamma}$. It can be seen that whether $\bar{\mu}_{i}=(1 \pm j \sqrt{-\gamma}) \mu_{i \pm}$ have negative real parts is decided by angle of $\bar{\mu}_{i}$. Note that phase angle of $\mu_{i+}$ is in $\left(90^{\circ}, 180^{\circ}\right)$ while that of $\mu_{i-}$ is in $\left(180^{\circ}, 270^{\circ}\right)$, and if $\sqrt{-\gamma}>0$, then $0<\theta_{\Gamma}=\arctan (\sqrt{-\gamma})<90^{\circ}$, so phase angles of $(1+j \sqrt{-\gamma}) \mu_{i+}$ and $(1-j \sqrt{-\gamma}) \mu_{i-}$ are in range of $\left(90^{\circ}, 270^{\circ}\right)$. In order to ensure all eigenvalues of $\Gamma$ have negative real parts, phase angles of $(1-j \sqrt{-\gamma}) \mu_{i+}$ and $(1+j \sqrt{-\gamma}) \mu_{i-}$ must be in range of $\left(90^{\circ}, 180^{\circ}\right)$ and $\left(180^{\circ}, 270^{\circ}\right)$, respectively, which means $\theta_{\Gamma}<\theta_{i}$.

If there are more than one pair of conjugate complex eigenvalues, it is obvious that $\theta_{\Gamma}<\theta_{i}$ should satisfied for all pairs of conjugate complexes, so $\theta_{\Gamma}<\min _{\beta_{i} \neq 0} \theta_{i}$. Note that $\theta_{\Gamma}=\arctan (\sqrt{-\gamma})$ and $\theta_{i}=\arctan \left(\alpha_{i} / \beta_{i}\right)$; then,

$$
\begin{gathered}
\arctan (\sqrt{-\gamma})<\min _{\beta_{i} \neq 0}\left[\arctan \left(\frac{\alpha_{i}}{\beta_{i}}\right)\right] \\
\Longrightarrow \sqrt{-\gamma}<\min _{\beta_{i} \neq 0}\left(\frac{\alpha_{i}}{\beta_{i}}\right) \\
\Longrightarrow \gamma>-\min _{\beta_{i} \neq 0}\left(\frac{\alpha_{i}^{2}}{\beta_{i}^{2}}\right) .
\end{gathered}
$$

So $-\min _{\beta_{i} \neq 0}\left(\alpha_{i}^{2} / \beta_{i}^{2}\right)<\gamma<0$. And for the other real eigenvalues, $\alpha_{i}^{2} / \beta_{i}^{2}=\infty$, which means that $\min _{\mu_{i} \neq 0}\left(\alpha_{i}^{2} / \beta_{i}^{2}\right)=$ $\min _{\beta_{i} \neq 0}\left(\alpha_{i}^{2} / \beta_{i}^{2}\right)$. Moreover, if all nonzero eigenvalues are real, $-\min _{\mu_{i} \neq 0}\left(\alpha_{i}^{2} / \beta_{i}^{2}\right)<\gamma<0$ turns to $-\infty<\gamma<0$, which is equal to $\gamma<0$; it is obvious that conditions of Case 1 and Case 2 can be rewritten in the same form as $-\min _{\mu_{i} \neq 0}\left(\alpha_{i}^{2} / \beta_{i}^{2}\right)<\gamma<0$.

2.2. Convergence Speed. Convergence speed of system (1) is decided by eigenvalue with the smallest absolute value of real part. Reconsider (12); if all nonzero eigenvalues have negative real parts, then the slowest convergence speed of system (2) is decided by the eigenvalue whose real part is the nearest to $Y$-axis. Three cases are analyzed according to choice of $\gamma$.

Case $1(0 \leq \gamma<1)$. From (7), it is obviously that the smallest eigenvalue is one of $\left((1-\sqrt{\lambda}) \mu_{i}\right)$, so smaller $1-\sqrt{\gamma}$ (or bigger $\gamma$ ) will cause slower convergence speed.

Case $2\left(-\min _{\mu_{i} \neq 0}\left(\alpha_{i}^{2} / \beta_{i}^{2}\right)<\gamma<0\right.$ and all nonzero $\mu_{i}$ are real). From (7), it is obvious that $\operatorname{Re}\left(\bar{\mu}_{i}\right)=\operatorname{Re}\left((1 \pm j \sqrt{-\gamma}) \mu_{i}\right)=\mu_{i}$, which means $\gamma$ has no effect on convergence speed. The bigger 


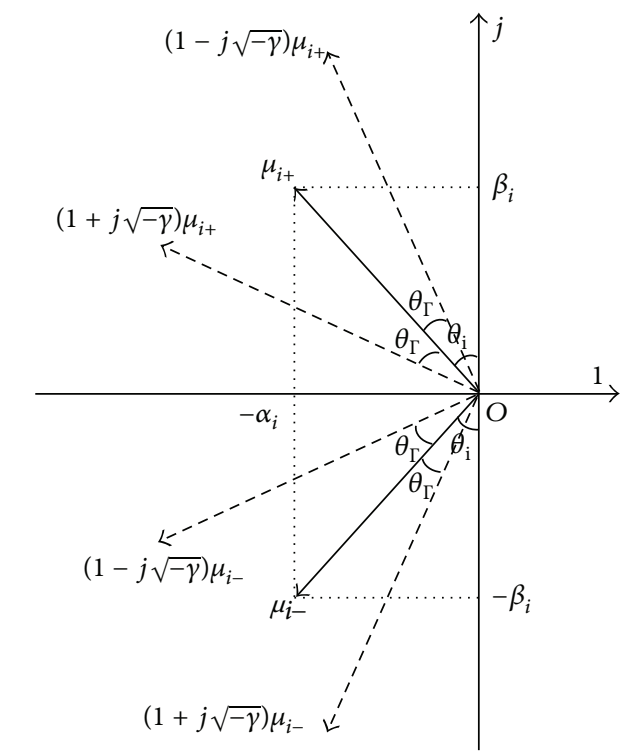

FIGURE 1: All possible results of $\bar{\mu}_{i}=(1 \pm j \sqrt{-\gamma}) \mu_{i}$.

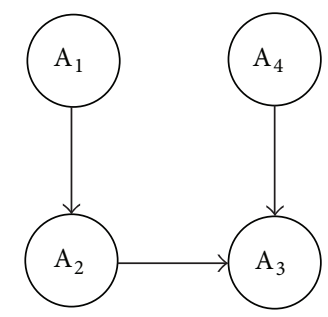

Figure 2: A digraph in [4].

imaginary part $\sqrt{-\gamma}$ of $\bar{\mu}_{i}$ means bigger $|\gamma|$ will cause larger amplitude of oscillation.

Case $3\left(-\min _{\mu_{i} \neq 0}\left(\alpha_{i}^{2} / \beta_{i}^{2}\right)<\gamma<0\right.$ and there are conjugate complex eigenvalues of $\left.\mu_{i}\right)$. From Figure $1, \operatorname{Re}\left(\bar{\mu}_{i}\right)$ is given by $\operatorname{Re}\left(\bar{\mu}_{i}\right)=\operatorname{Re}\left[(1-j \sqrt{-\gamma}) \mu_{i+}\right]=\operatorname{Re}[(1-j \sqrt{-\gamma})(-\alpha+j \beta)]=$ $\beta \sqrt{-\gamma}-\alpha$, so bigger $\beta \sqrt{-\gamma}-\alpha$ (or smaller $\gamma$ ) will cause slower convergence.

\section{Simulation Results}

In this section, examples are illustrated to prove conclusions drawn in Section 2.

\subsection{Case 1. A digraph in [4] is shown in Figure 2.}

It is obvious that $-L=\left[\begin{array}{cccc}-1 & 1 & 0 & 0 \\ 0 & -1 & 1 & 0 \\ 0 & 0 & 0 & 0 \\ 0 & 0 & 1 & -1\end{array}\right]$, whose eigenvalues are $\lambda_{1}=0$ and $\lambda_{2,3,4}=-1$. Choose initial coordinate of the four agents as $x_{0}=\left[\begin{array}{llll}0.6 & -0.4 & -0.2 & 0.8\end{array}\right]^{T}$ and $y_{0}=$ $\left[\begin{array}{llll}-0.8 & 0.7 & -0.9 & -1\end{array}\right]^{T}$, respectively. All simulation results are shown in Figure 3 according to different $\gamma>0$.

From Figure 3, asymptotical consensus is achieved when $0 \leq \gamma<1$, while it is not achieved if $\gamma \geq 1$. And agents stay in fixed positions when $\gamma=1$ while they reflect in separating trend.
Moreover, asymptotical consensus is achieved in less than 10 seconds when $\gamma=0$ and in about 20 seconds when $\gamma=0.5$, which agrees with Case 1 in Section 2.2.

3.2. Case 2. Simulation results with the same system when $\gamma<0$ are shown in Figure 4.

If all eigenvalues of $-L$ are real, any $\gamma<0$ can satisfy Lemma 2, so consensuses are achieved when $\gamma=-0.5$ or $\gamma=-3$. There are no obvious differences between their convergence speeds. More oscillation appears when $\gamma=-3$, which agrees with Case 2 in Section 2.2.

3.3. Case 3. Next, $-L$ is chosen as $\left[\begin{array}{cccc}-2 & 1 & 0 & 1 \\ 0 & -1 & 1 & 0 \\ 1 & 0 & -1 & 0 \\ 0 & 0 & 0 & 0\end{array}\right]$, and other conditions remain the same, so eigenvalues are $\lambda_{1}=0$, $\lambda_{2}=-0.2451$, and one pair of conjugate complex eigenvalues $\lambda_{3,4}=-1.8774 \pm j 0.7449$. Simulation results are shown in Figure 5.

According to Lemma $2,-\min _{\mu_{i} \neq 0}\left(\alpha_{i}^{2} / \beta_{i}^{2}\right)=-6.3521$. When $-\min _{\mu_{i} \neq 0}\left(\alpha_{i}^{2} / \beta_{i}^{2}\right)<\gamma=-1<0$ or $-\min _{\mu_{i} \neq 0}\left(\alpha_{i}^{2} / \beta_{i}^{2}\right)<$ $\gamma=-3<0$, consensus is achieved, while opposite result is obtained when $\gamma=-7<-\min _{\mu_{i} \neq 0}\left(\alpha_{i}^{2} / \beta_{i}^{2}\right)$. And the convergence speed is a little faster when $\gamma=-1$, which agrees with Case 3 in Section 2.2. 

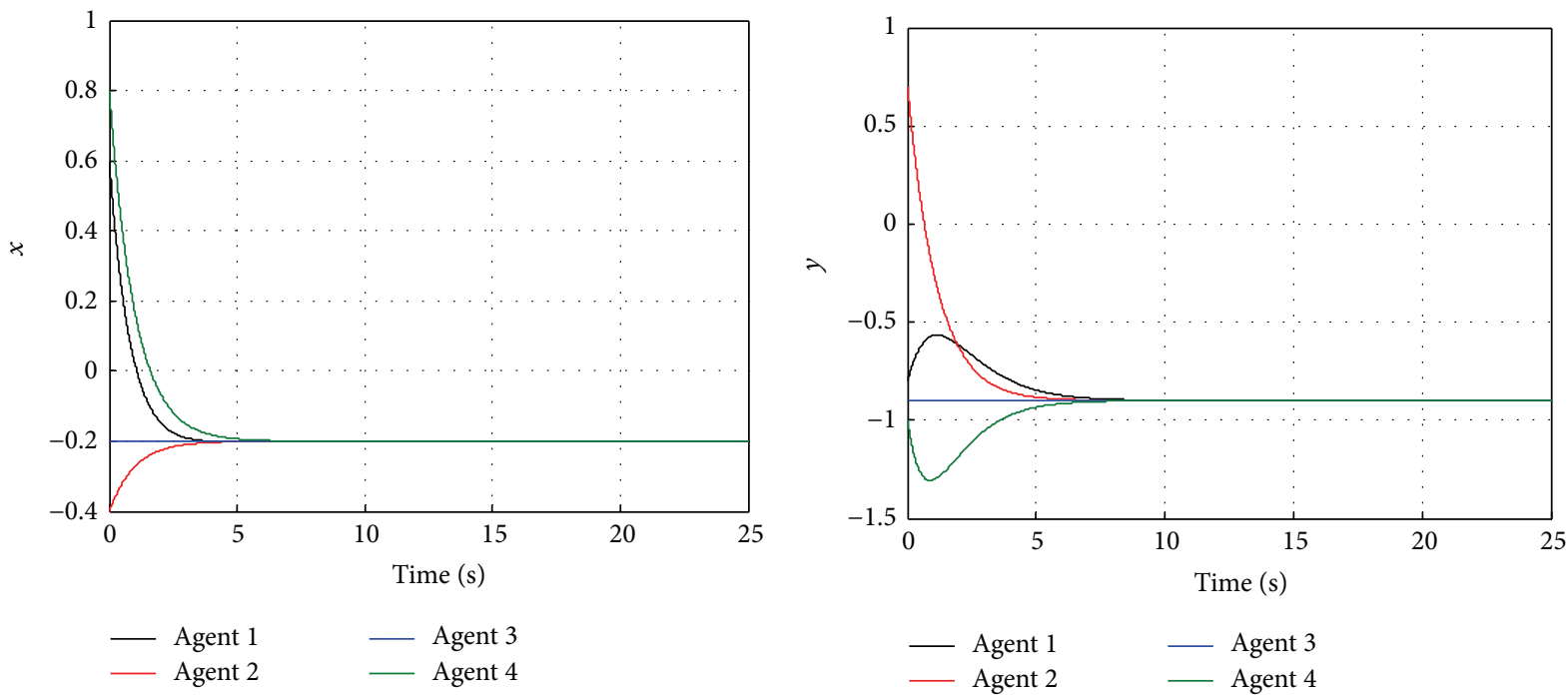

(a) Simulation results $(\gamma=0)$
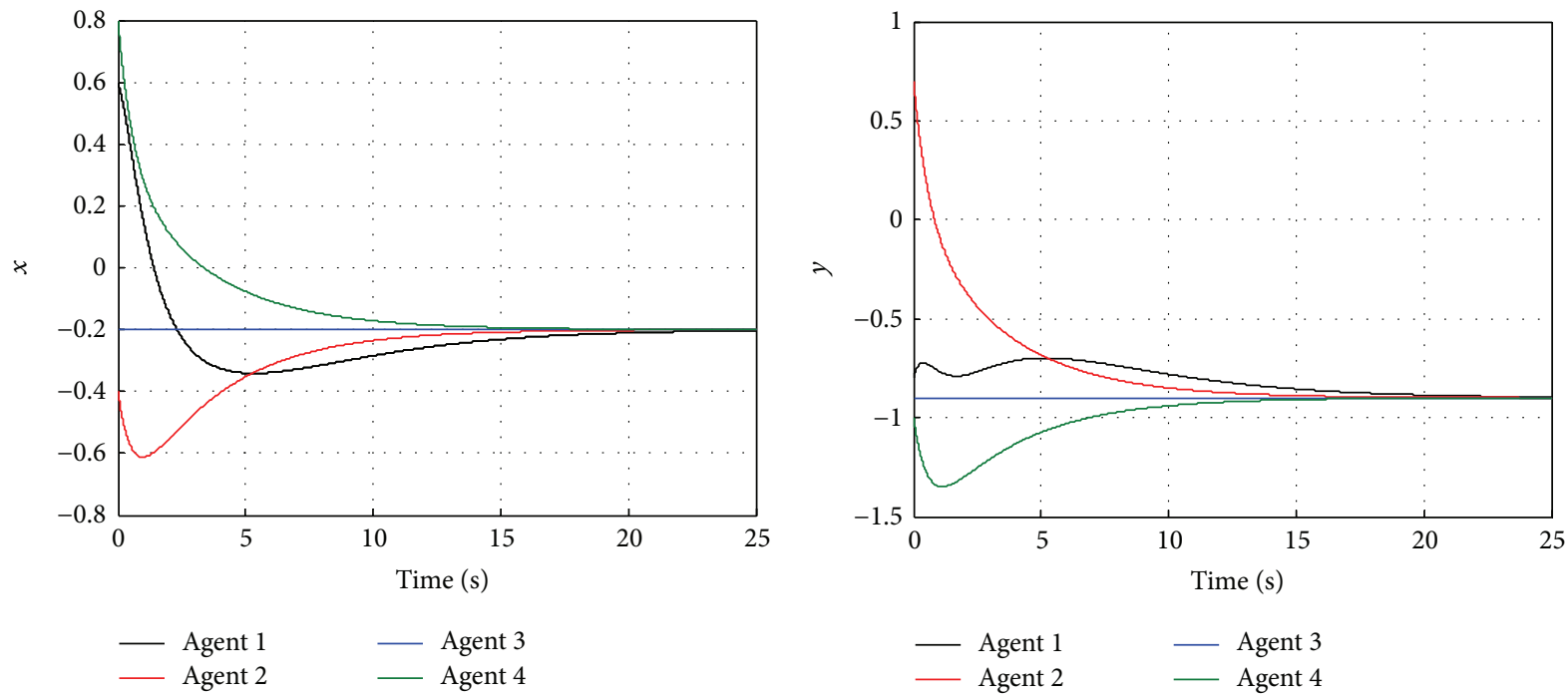

(b) Simulation results $(\gamma=0.5)$
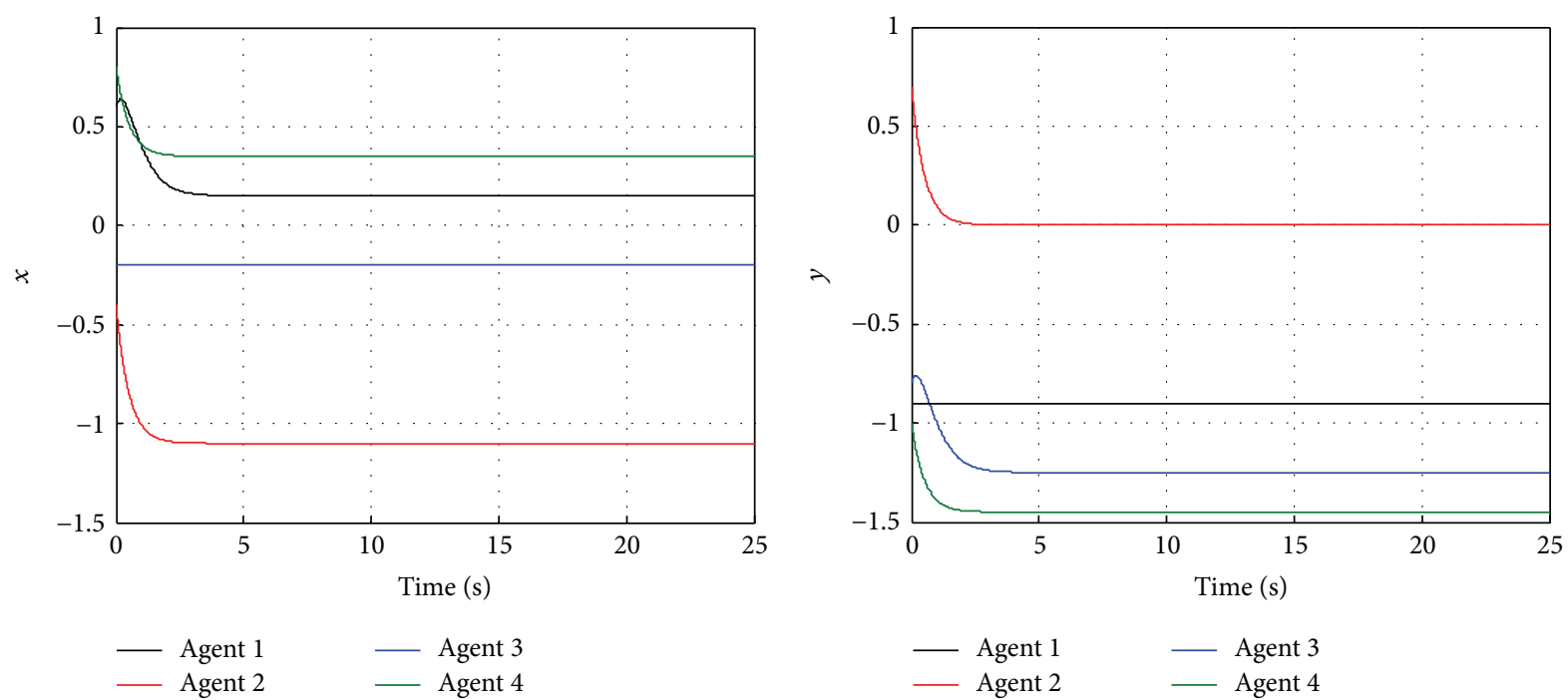

$\begin{array}{rr}\text { Agent } 1 & \text { Agent } 3 \\ - \text { Agent 2 } & \text { Agent 4 }\end{array}$

(c) Simulation results $(\gamma=1)$

Figure 3: Continued. 

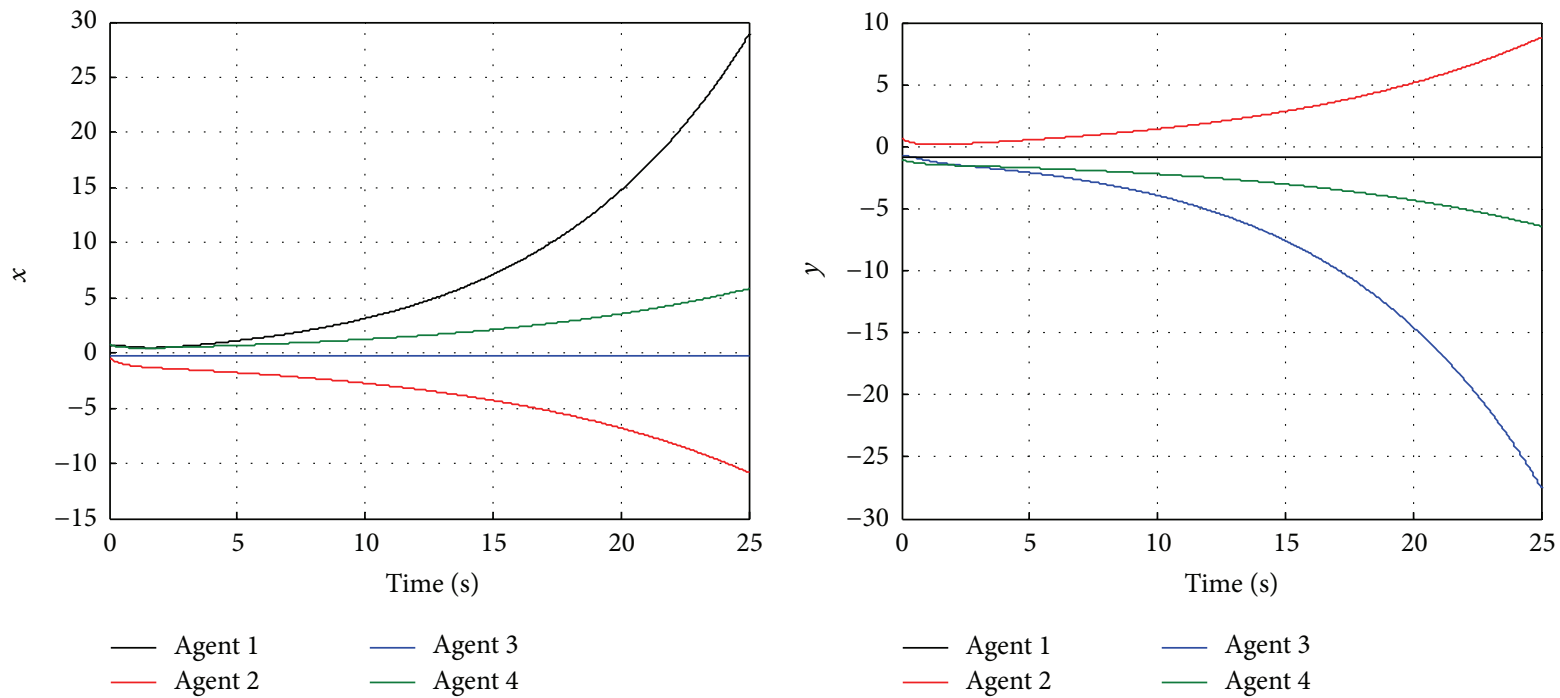

(d) Simulation results $(\gamma=1.2)$

FigURE 3: Simulation results with different $\gamma>0$.
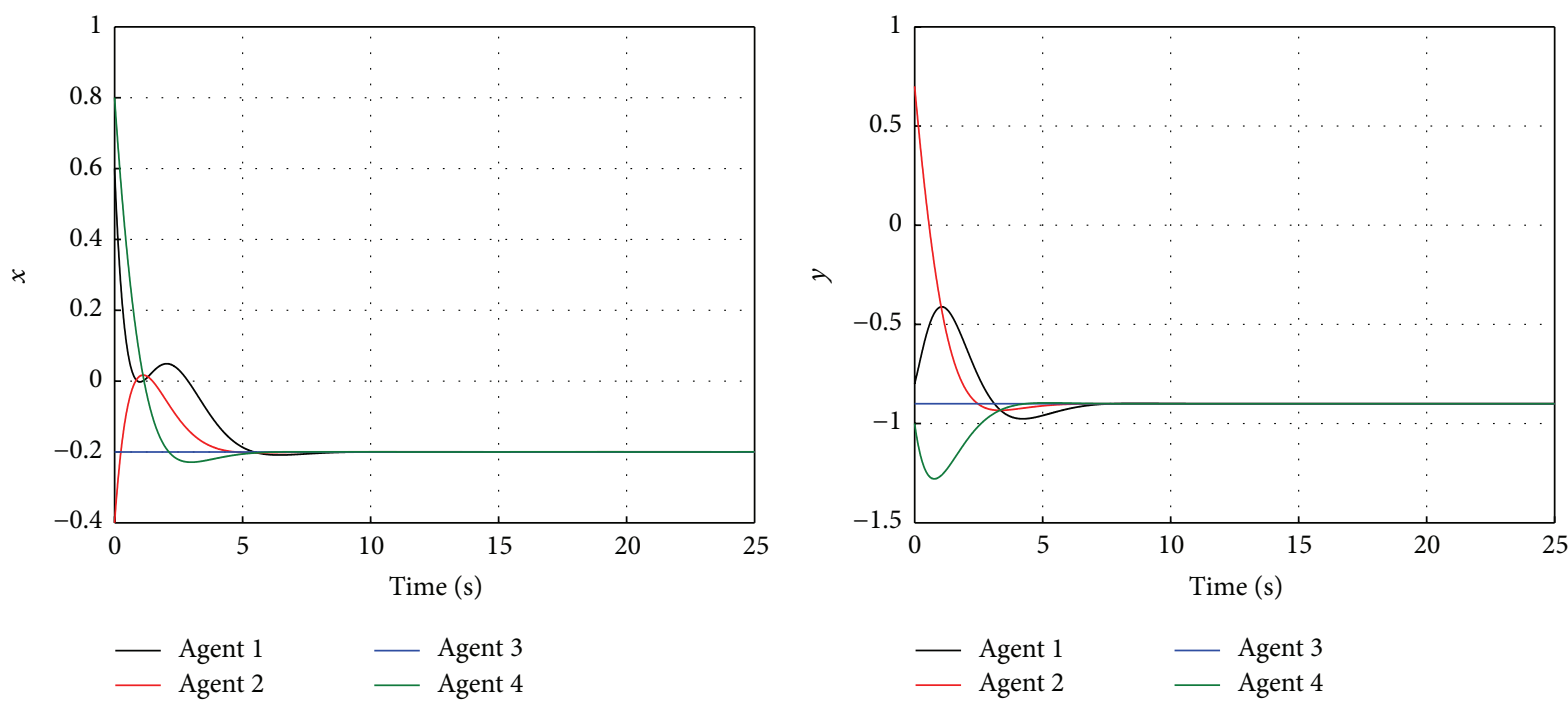

(a) Simulation results $(\gamma=-0.5)$
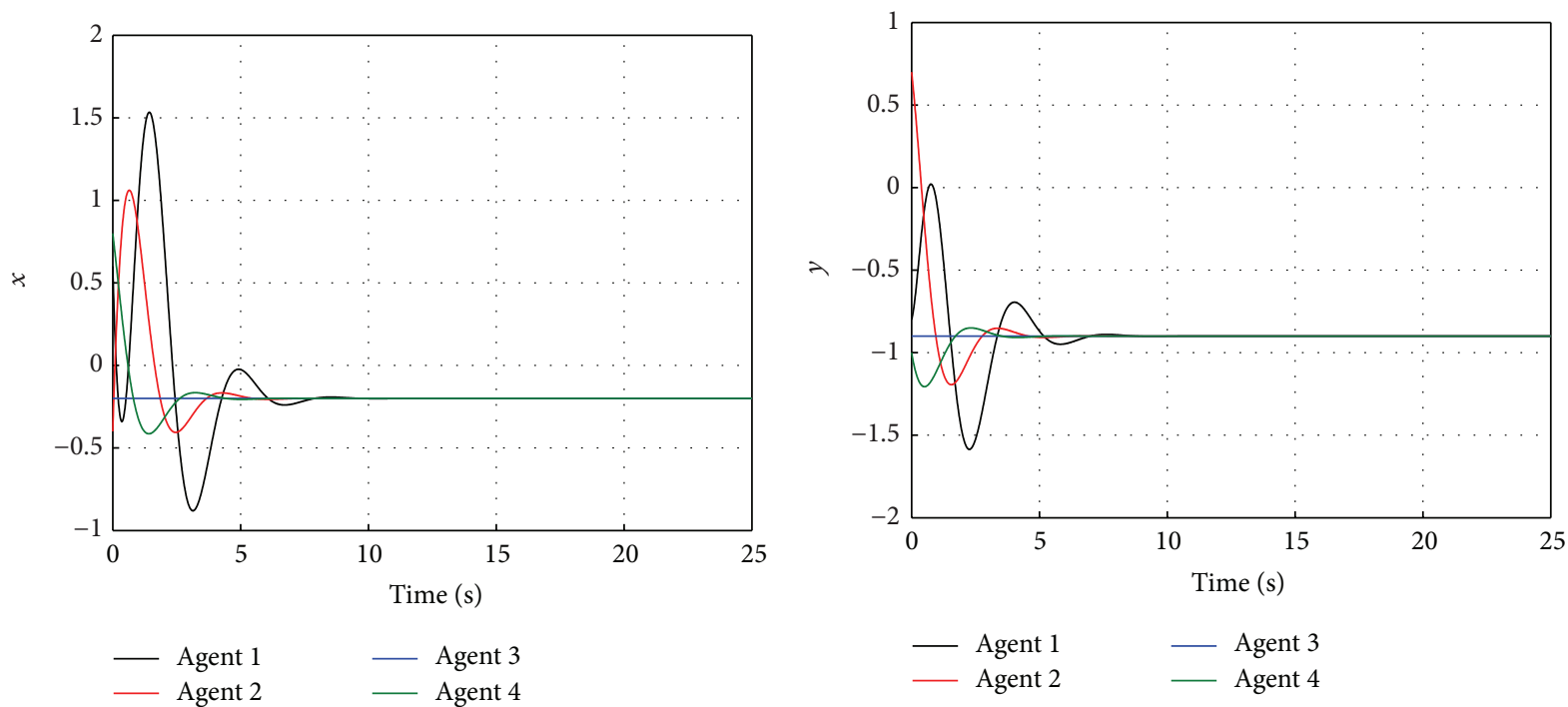

(b) Simulation results $(\gamma=-3)$

Figure 4: Simulation results with different $\gamma<0$ when all eigenvalues of $-L$ is real. 


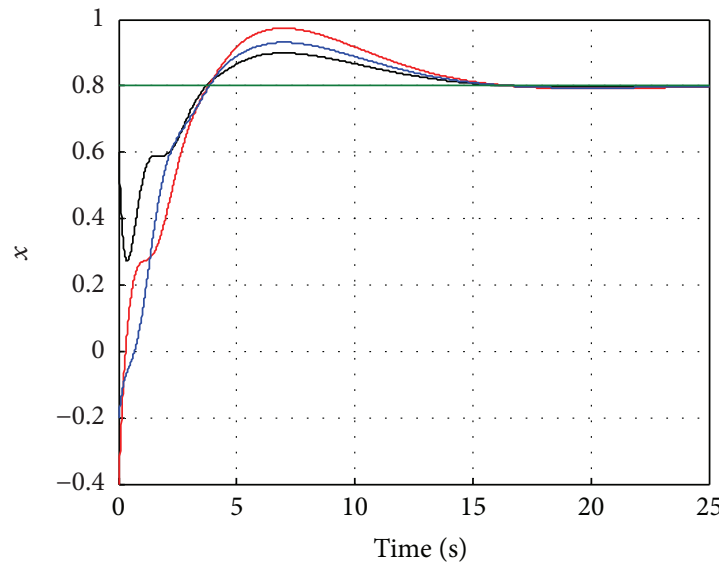

$\begin{array}{ll}\text { Agent } 1 & \text { - Agent } 3 \\ \text { Agent } 2 & \text { Agent } 4\end{array}$

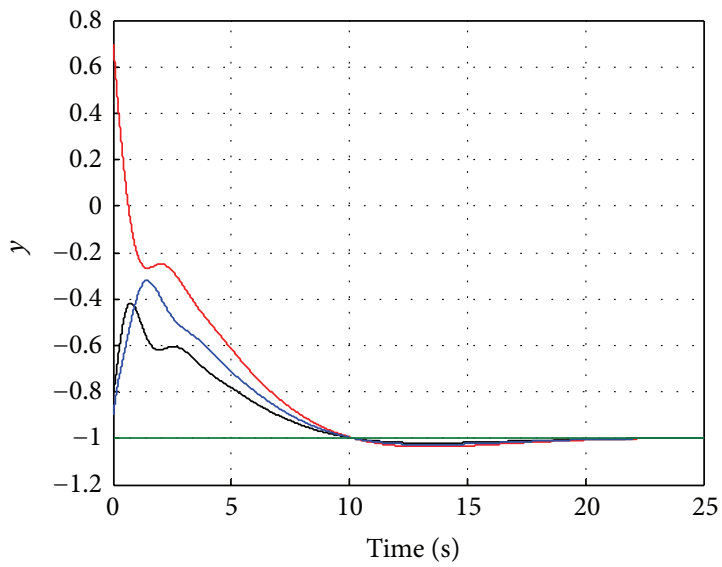

- Agent $1 \quad$ Agent 3

(a) Simulation results $(\gamma=-1)$
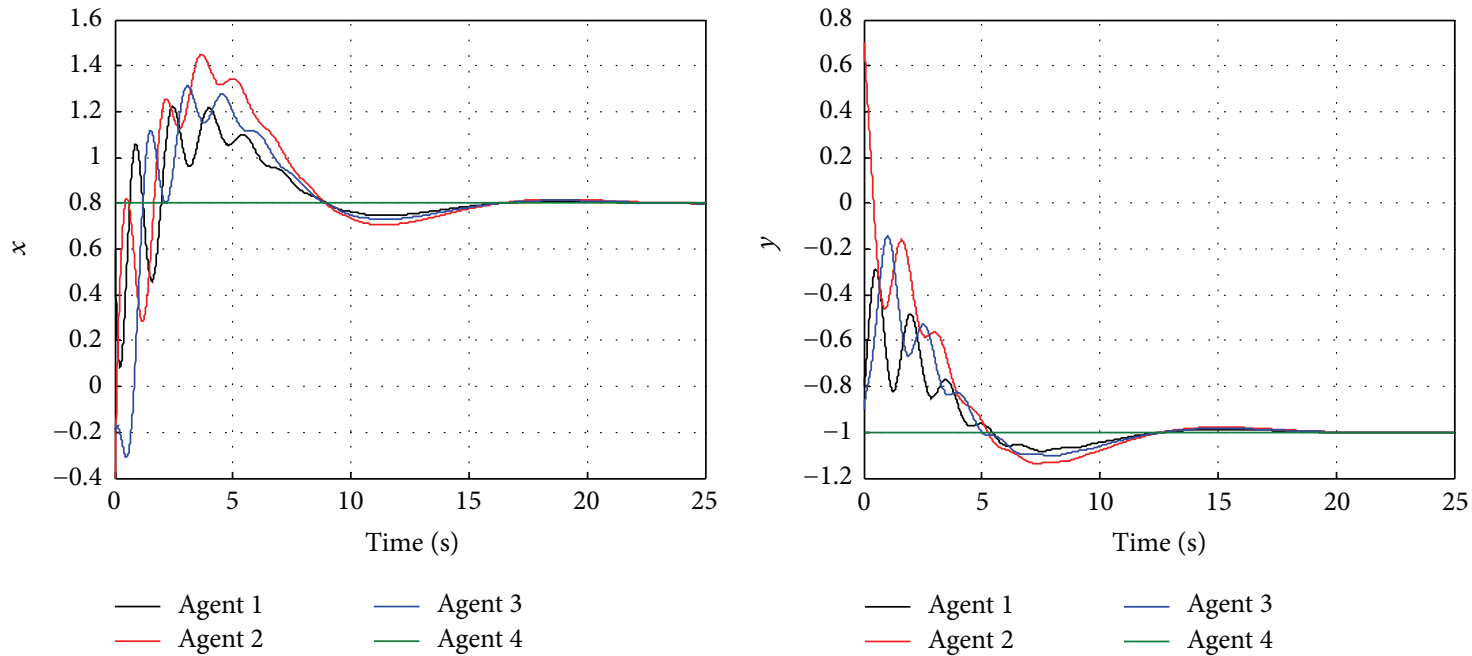

(b) Simulation results $(\gamma=-3)$
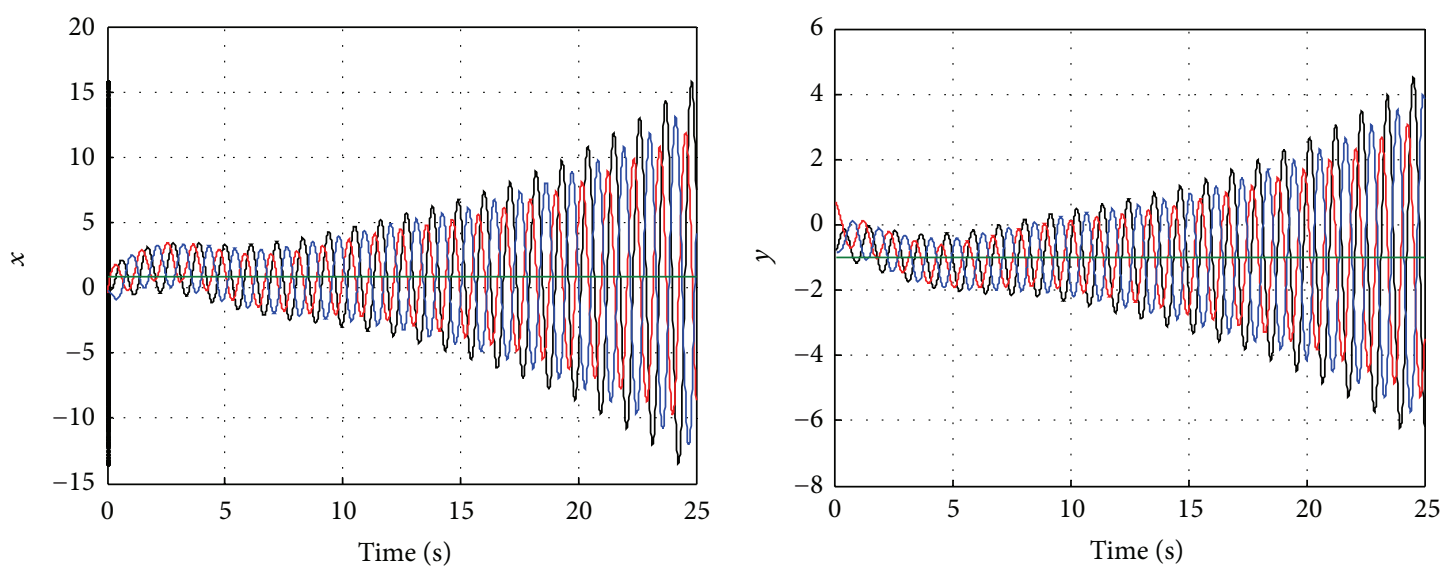

Agent 1
- Agent 2
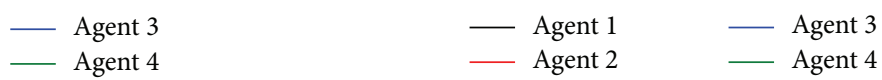

(c) Simulation results $(\gamma=-7)$

FIGURE 5: Simulation results with different $\gamma<0$ when there are conjugate complex eigenvalues. 


\section{Conclusion}

A novel consensus protocol is presented for the linear twodimension first-order multiagent systems (MASs). Numerical examples illustrated the effectiveness of the proposed consensus protocol according to different key parameters. Simulation results ensure the relationship between range of key parameter and convergence speed.

\section{Conflict of Interests}

The authors declare that there is no conflict of interests regarding the publication of this paper.

\section{Acknowledgments}

This work is supported by National Natural Science Foundation of China (nos. 61374083 and 61203177) and Zhejiang Province Key Project of Science and Technology (no. 2014C03027).

\section{References}

[1] D. Zhang, W. J. Cai, L. H. Xie, and Q. G. Wang, "Non-fragile distributed filtering for T-S fuzzy systems in sensor networks," IEEE Transactions on Fuzzy Systems, 2014.

[2] Y. Yi, Z. Y. Lin, and G. F. Yan, "Variable speed running on kneed biped robot with underactuation degree two," International Journal of Humanoid Robotics, vol. 11, Article ID 1450015, 2014.

[3] A. Jadbabaie, J. Lin, and A. S. Morse, "Coordination of groups of mobile autonomous agents using nearest neighbor rules," in Proceedings of the 41st IEEE Conference on Decision and Control, pp. 2953-2958, December 2002.

[4] Z. Y. Lin, L. L. Wang, Z. M. Han, and M. Y. Fu, "Distributed formation control of multi-agent systems using complex laplacian," IEEE Transactions on Automatic Control, vol. 59, no. 7, pp. 1765-1777, 2014.

[5] C.-Q. Ma and J.-F. Zhang, "Necessary and sufficient conditions for consensusability of linear multi-agent systems," IEEE Transactions on Automatic Control, vol. 55, no. 5, pp. 1263-1268, 2010.

[6] H. Huang, C. B. Yu, and Q. H. Wu, "Autonomous scale control of multiagent formations with only shape constraints," International Journal of Robust and Nonlinear Control, vol. 23, no. 7, pp. 765-791, 2013.

[7] Z. K. Li, X. D. Liu, W. Ren, and L. H. Xie, "Distributed tracking control for linear multiagent systems with a leader of bounded unknown input," IEEE Transactions on Automatic Control, vol. 58, no. 2, pp. 518-523, 2013.

[8] H.-W. Yu and Y.-F. Zheng, "Dynamic behavior of multi-agent systems with distributed sampled control," Acta Automatica Sinica, vol. 38, no. 3, pp. 357-365, 2012.

[9] Y.-Y. Chen and Y.-P. Tian, "Directed coordinated control for multi-agent formation motion on a set of given curves," Acta Automatica Sinica, vol. 35, no. 12, pp. 1541-1549, 2009. 


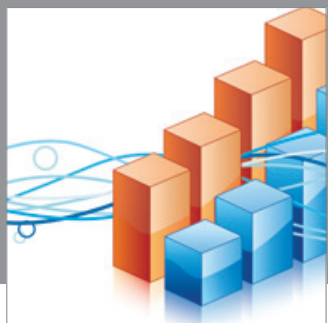

Advances in

Operations Research

mansans

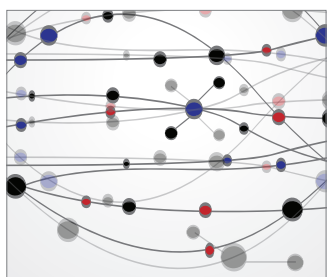

The Scientific World Journal
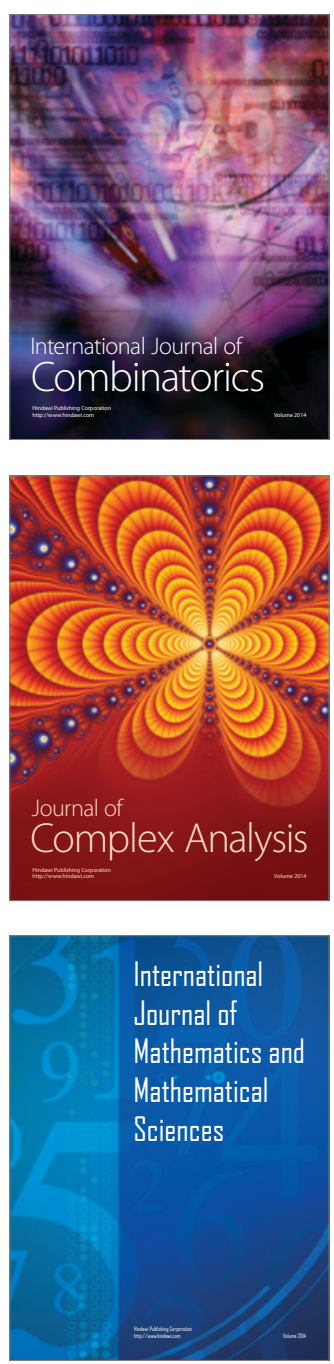
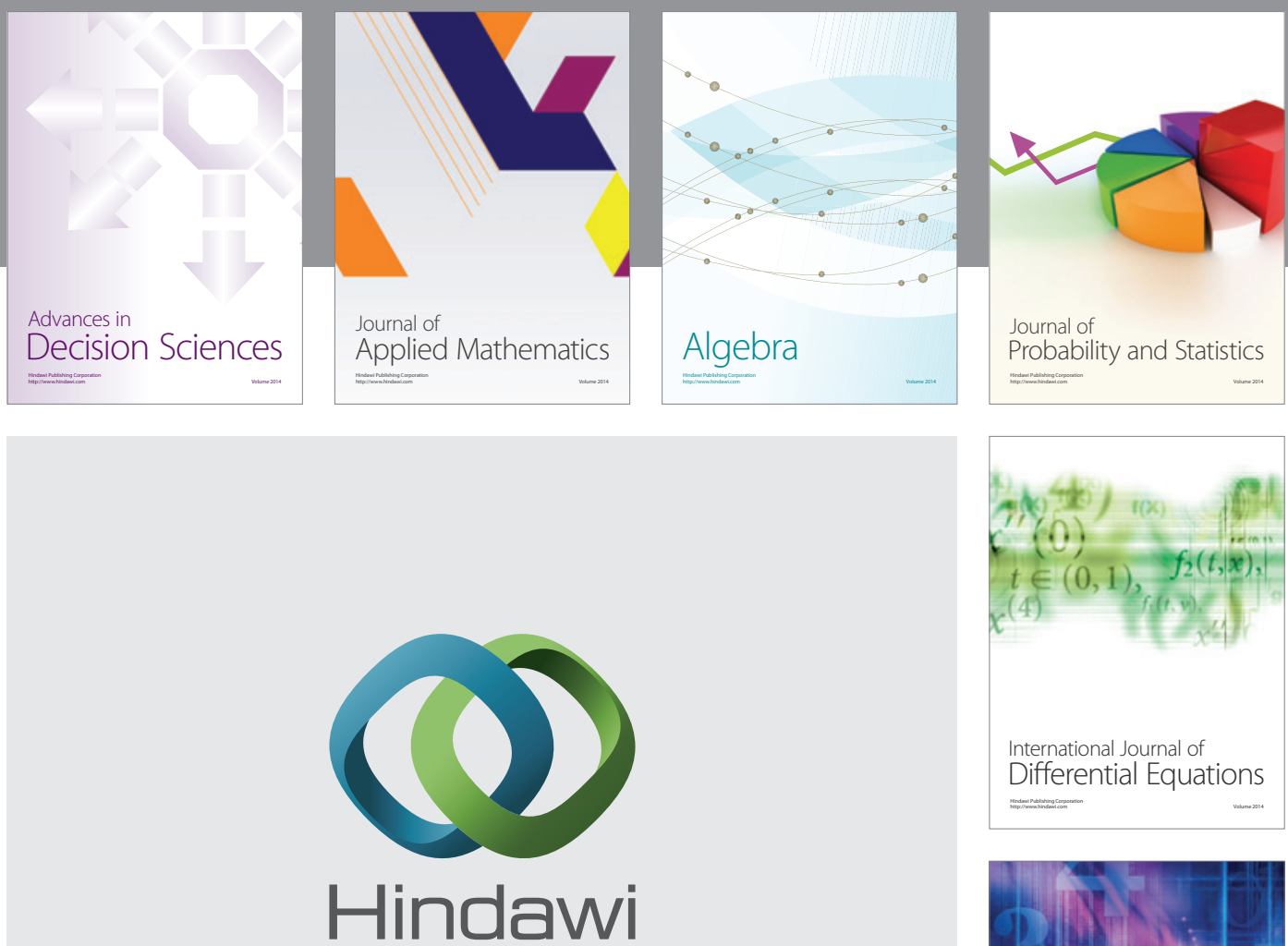

Submit your manuscripts at http://www.hindawi.com
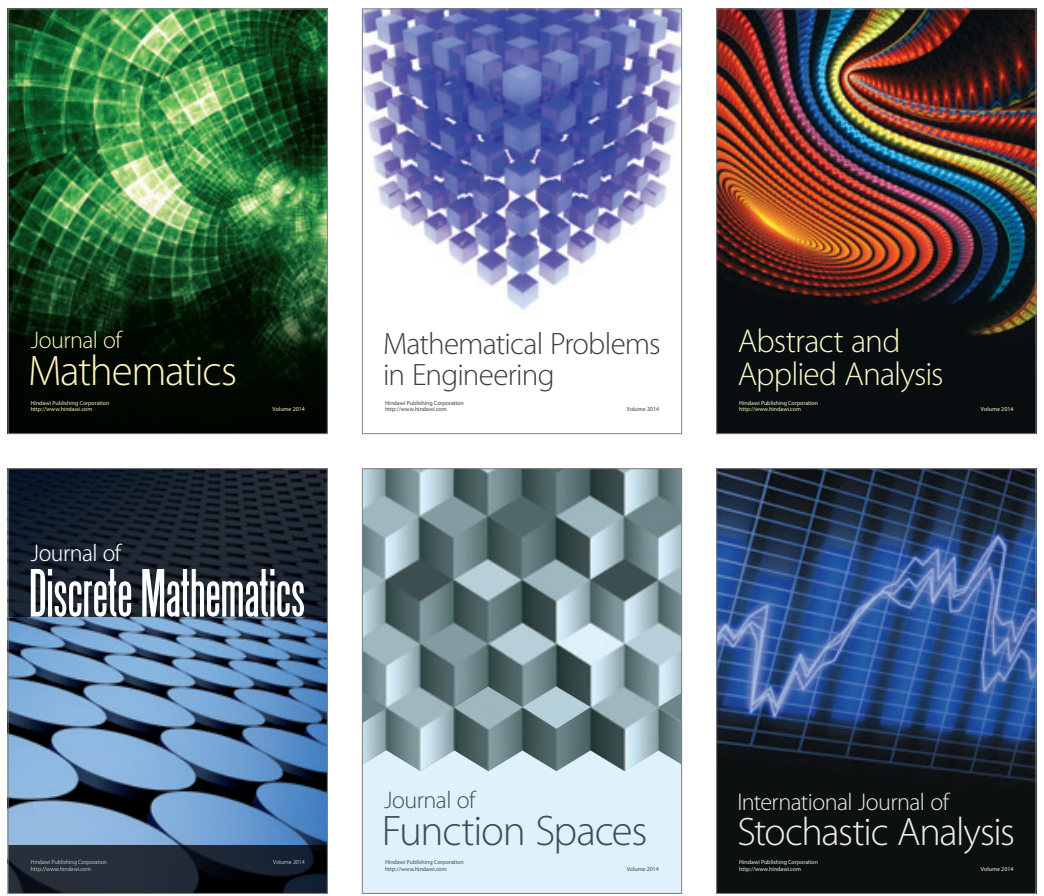

Journal of

Function Spaces

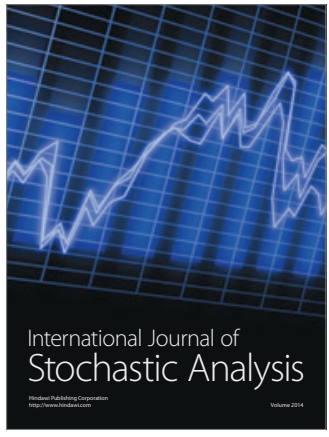

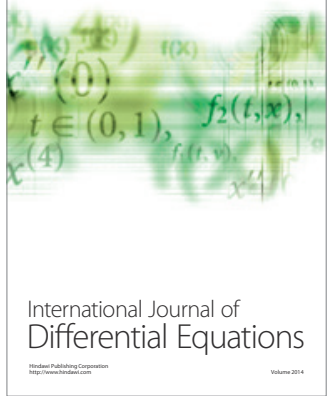
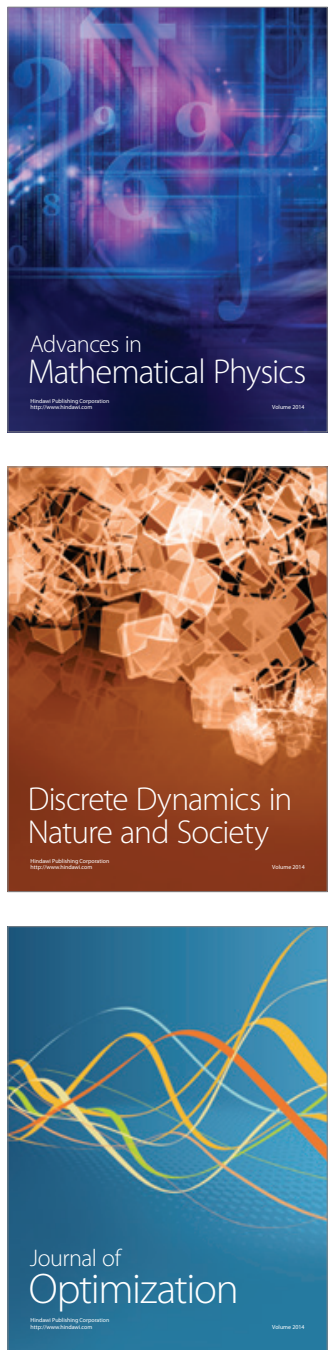